\title{
Quantitative aspects of patello-femoral cartilage fibrillation in Liverpool necropsies
}

\author{
G. MEACHIM AND I. H. EMERY* \\ Department of Pathology, University of Liverpool, and Department of Orthopaedics, \\ Walton Hospital, Liverpool
}

The significance of ageing as a possible factor contributing to the pathogenesis of osteoarthrosis is debatable (Byers, Contepomi, and Farkas, 1970); moreover, the matter may require separate consideration for the hip, knee, and other joints. Necropsy studies of the incidence, natural history, and severity of age-related cartilage lesions in various joints are relevant to this question. Age changes in the hip, knee, and other joints have been studied by Heine (1926), in the knee joint by Øwre (1936) and Bennett, Waine, and Bauer (1942), in the elbow by Goodfellow and Bullough (1967), and in the hip by Byers and others (1970). These previous studies have been based mainly on a qualitative assessment of the state of the articular surfaces, the results being expressed, for example, in terms of a grading system such as that devised by Collins (1949). The present study, in contrast, is based on a quantitative method in which the results are expressed as a percentage of the articular surface area affected by destructive cartilage changes. The patello-femoral component of the knee joint was selected for study by this method, since the slight curvature of the patellar surface makes this articulation suitable for quantitation by a point-counting technique. Previous studies, with the exception of that by Goodfellow and Bullough (1967), mostly seem to have relied mainly on naked-eye inspection of unstained surfaces. The present study, in contrast, was made on surfaces which had been painted with indian ink, since such preparations make cartilage lesions more readily apparent (Bullough and Goodfellow, 1968; Meachim, 1972a) and facilitate point-counting of the various types of surface change. Naked-eye examination was supplemented by stereomicroscopy of the surface and, where indicated, by transmitted light microscopy of tangential surface slices (Meachim, 1972a).

\section{Material and methods}

The study was made on the patello-femoral articulation of one or both knee joints from a random series of necropsies within the city of Liverpool on 98 white subjects
(53 male; 45 female) whose ages ranged from 4 weeks to 94 years. Persons dying from accidents and persons dying outside hospital suddenly or unexpectedly from natural causes were included as far as possible. Joints showing evidence of previous inflammatory disease, disuse atrophy, surgery such as meniscectomy, old injury, or injury from a recent accident were excluded. Quantitative data were obtained from indian ink preparations of the left patellar articular surface in all of the 98 subjects, from the opposing left femoral articular surface in 83 of them (43 male; 40 female), and also from the contralateral right patellar surface in thirty of the adults (14 men and 16 women).

\section{SURFACE QUANTITATION}

Indian ink preparations were made by the method previously described (Meachim, 1972a). The painted articular surfaces were then examined by the unaided naked eye, by study of photographic prints at a magnification of $\times 2$, by stereomicroscopy en face at a magnification of $\times 10$, and, where indicated, by transmitted light microscopy of tangential surface slices.

The appearance at each site on the painted surface was noted as follows:

(1) Sites at which no distinct markings were seen;

(2) Sites showing dark ink markings against a pale grey background, such marking having either approximately parallel alignments or other, more complex, configurations on macroscopic examination;

(3) Sites showing confluent or semi-confluent blackening;

(4) Sites of full-thickness loss of the original cartilage.

For each of the above appearances, the percentage it occupied of the total articular surface area was determined by a point-counting technique. For counting, a grid with points arrayed at the corners of squares $5 \mathrm{~mm} .^{2}$ in area was superimposed over photographic prints taken with a Polaroid Land camera set at a magnification of $\times 2$. Since the patellar and the opposing femoral surfaces are curved, their medial and lateral portions were each photographed separately en face and montages were made from the pairs of prints.

The patellar counts were made from over the whole of the patellar articular surface visible en face. The opposing femoral counts were made from the femoral groove together with the proximal part of the convex region of the medial condyle at the level of the intercondylar notch, since there is evidence that the patella comes into contact 
with this part of the condyle on full flexion of the knee (Last, 1972; Emery, unpublished observations). The distal border for the femoral point-counting area was taken as follows. On the lateral condyle there is a curving ridge which was taken as the distal border on the lateral side (Walmsley, 1972). On the medial condyle, the area counted extended down to a horizontal line level with the lowest point of the curved ridge on the lateral condyle; the choice of this medial distal border was arbitrary, since, except in adolescents, there are no medial anatomical landmarks to indicate the distal limit of patellofemoral contact on the medial femoral condyle.

All the specimens from the left knee joint were examined unfixed, and were painted either immediately after removal or after storage in physiological saline at $5^{\circ} \mathrm{C}$. for periods of up to 3 days. In the case of the right patellae, the appearance in indian ink preparations was compared between seventeen specimens painted without previous fixation and thirteen specimens painted after first being placed in buffered formol-saline for periods of up to 7 days. This comparison showed that fixation had no obvious detrimental effect on the subsequent demonstration of sites showing confluent or semi-confluent blackening. However, it did seem slightly to impede the detection of sites showing dark ink markings against a pale grey background; the quantitative data obtained for such markings on the right patellae were therefore excluded from the final analysis of the results.

\section{HISTOLOGY}

Vertically-cut blocks for histology of paraffin-embedded sections were taken from the upper, medial, lateral, and distal poles of nineteen of the adult patellae, from subjects ( 9 men; 10 women) whose ages ranged from 24 to 86 years. Histological sections were examined for the presence or absence of a fibrous covering layer over the periphery of the articular surface of the cartilage; only coverings sited where they would have been visible when the surface was examined en face were included in this assessment; fibrous covering adherent to a steeply-sloping non-articular side of the cartilage was excluded.

\section{Results}

\section{OVERT DESTRUCTIVE CHANGES}

In most of the specimens the appearance of the cartilage surface after painting with indian ink varied from site to site (Fig. 1). The quantitative results will first be presented only for those sites showing confluent or semi-confluent blackening (Figs 1 and 2) and those showing full-thickness cartilage loss (Figs 3 and 4); sites showing dark markings against a pale grey background (Figs 1 and 2) will be considered separately in a later section. Confluent or semi-confluent blackening (Figs 1 and 2) was in most instances indicative of overt fibrillation of exposed cartilage (Meachim, 1972a); however, at the edge of the joint, it represented either fibrillation or a covering of the periphery of the cartilage surface by a layer of fibrous tissue, and sites of peripheral fibrous covering are therefore included in the surface percentages given in this section of the text and in its

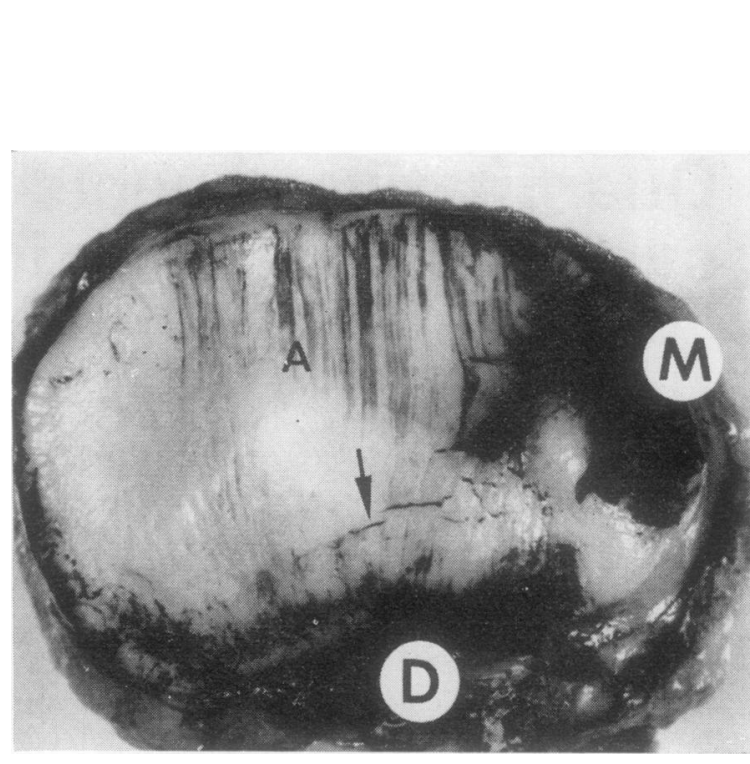

FIG. 1 Indian ink preparation of left patellar articular surface, viewed en face, from a woman aged 24 years, showing confluent and semi-confluent blackening on the medial part of the medial facet $(M)$ and on the periphery of the cartilage near the distal edge (D). Dark markings against a pale grey background $(A)$ extend in approximately parallel alignment across the surface from the proximal edge. Note also the transverse cracks (arrow)

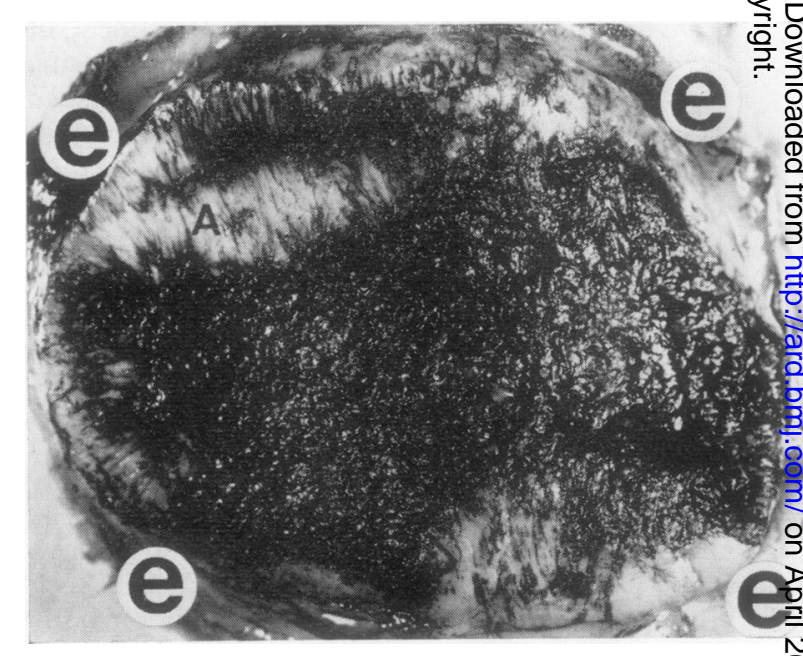

FIG. 2 Indian ink preparation of left patellar articular surface from a man aged 70 years. Most of the cartilage shows a confluent blackening; some areas showing dark markings against a pale grey background $(A)$ are also seen. Patellar edge (e)

accompanying figures. Occasionally a more central area of exposed cartilage on which the superficial layer had been destroyed showed less blackening than the expected amount, because the thinned area had a relatively smooth surface without the deep splitting usually seen in overt fibrillation. Sites of full-thickness loss of the original cartilage (Figs 3 and 4) showed a 


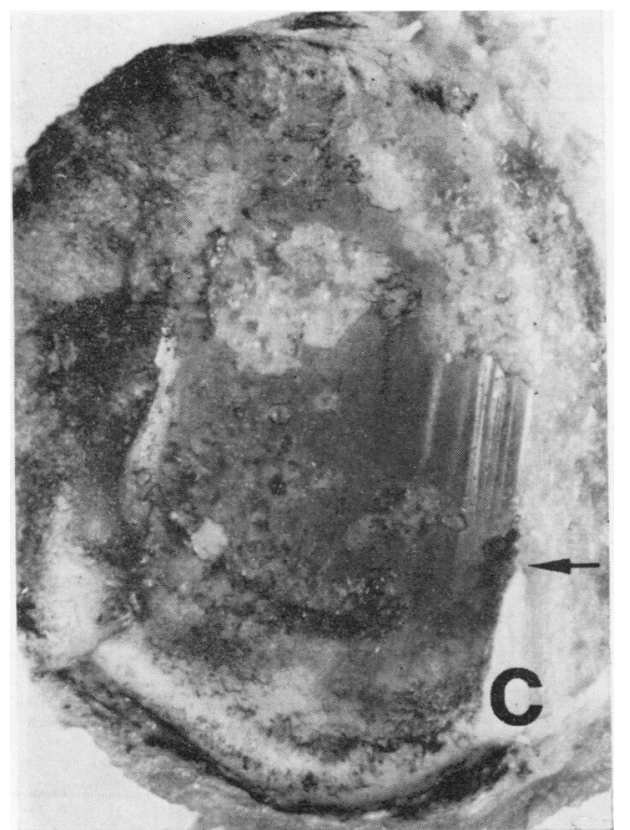

FIG. 3 Part of the left patellar surface from a woman aged 88 years, showing an area of full-thickness cartilage loss and bone exposure, with linear highlights. An angled vertical cut has been made through one side of the specimen: the cut face shows a wedge-shaped segment of old cartilage $(C)$ with destructive thinning. The apex of the wedge (arrow) abuts on the area of full-thickness cartilage loss and bone exposure

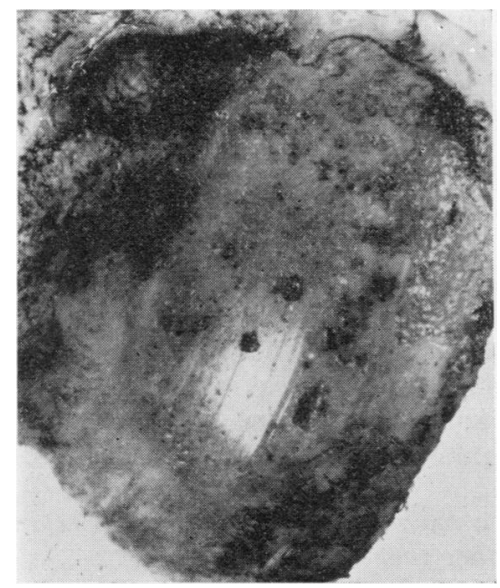

FIG. 4 Lateral aspect of the femoral surface opposing the patella illustrated in Fig. 3. There is a large area of fullthickness cartilage loss and bone exposure, with linear highlights

surface of exposed bone; the exposed bone was sometimes interspersed with plugs of new nonosseous tissue. On a few occasions 'central osteo- phytosis' was apparent, and such areas are included with the percentages given for full-thickness cartilage loss.

The individual results for the percentage of the articular surface area involved by overt destructive changes and peripheral fibrous covering are depicted for the left patello-femoral joint in Figs 5, 6, and 7 (overleaf).

The means of these results calculated by agedecade, separately for each sex, are shown in Fig. 8 (overleaf).

It will be appreciated that point-counting figures obtained from subjects in the age-decade 0 to 9 years were not strictly comparable with those from teenagers and adults, because of the smaller total area of the joint surfaces in childhood, and because of the possibility that immaturity of the articular and juxta-articular surface might in itself affect the appearance on painting with indian ink. In the case of the later age-decades, the results (Figs 5, 6, and 8) show that overt destructive changes at the patello-femoral articulation were extremely common amongst adults, and that they were often also apparent in teenagers.

Within each age-decade there was variation between individuals (Figs 5 and 6) in the percentage area of overt fibrillation and peripheral fibrous covering and, for older subjects, in the percentage area of full-thickness cartilage loss and in the presence or absence of osteophytosis at the articular margin. For technical reasons it was not practicable to study total body weight as a possible influence on this individual variation. It was also impracticable to obtain occupational histories (Kellgren, 1961); for geographical reasons it is likely that few or none of the men had been coal-miners.

The results according to age (Figs 5, 6, and 8) indicate that overt destructive change, subject to the variation from individual to individual in its rate of development, sooner or later involved virtually the whole of the patellar and, particularly in women, a considerable proportion of the opposing femoral surface. The average rate of development was relatively slow in terms of the expected total life-span of an individual: amongst the Liverpool population it can be estimated that on average approximately 50 per cent. of the male and 55 per cent. of the female left patellar surface, and approximately 30 per cent. of the male and 40 per cent. of the female opposing left femoral surface, had developed overt damage by the age of 50 years (Fig. 8). Particularly after the age of 50 years, the percentage area affected tended to be greater in women than in men, especially on the femoral surface (Figs 5, 6, and 8); sex differences in the point-counting results may perhaps be partly related to the fact that the total area of the patellar articular surface was on average smaller in the women (Table, overleaf). 


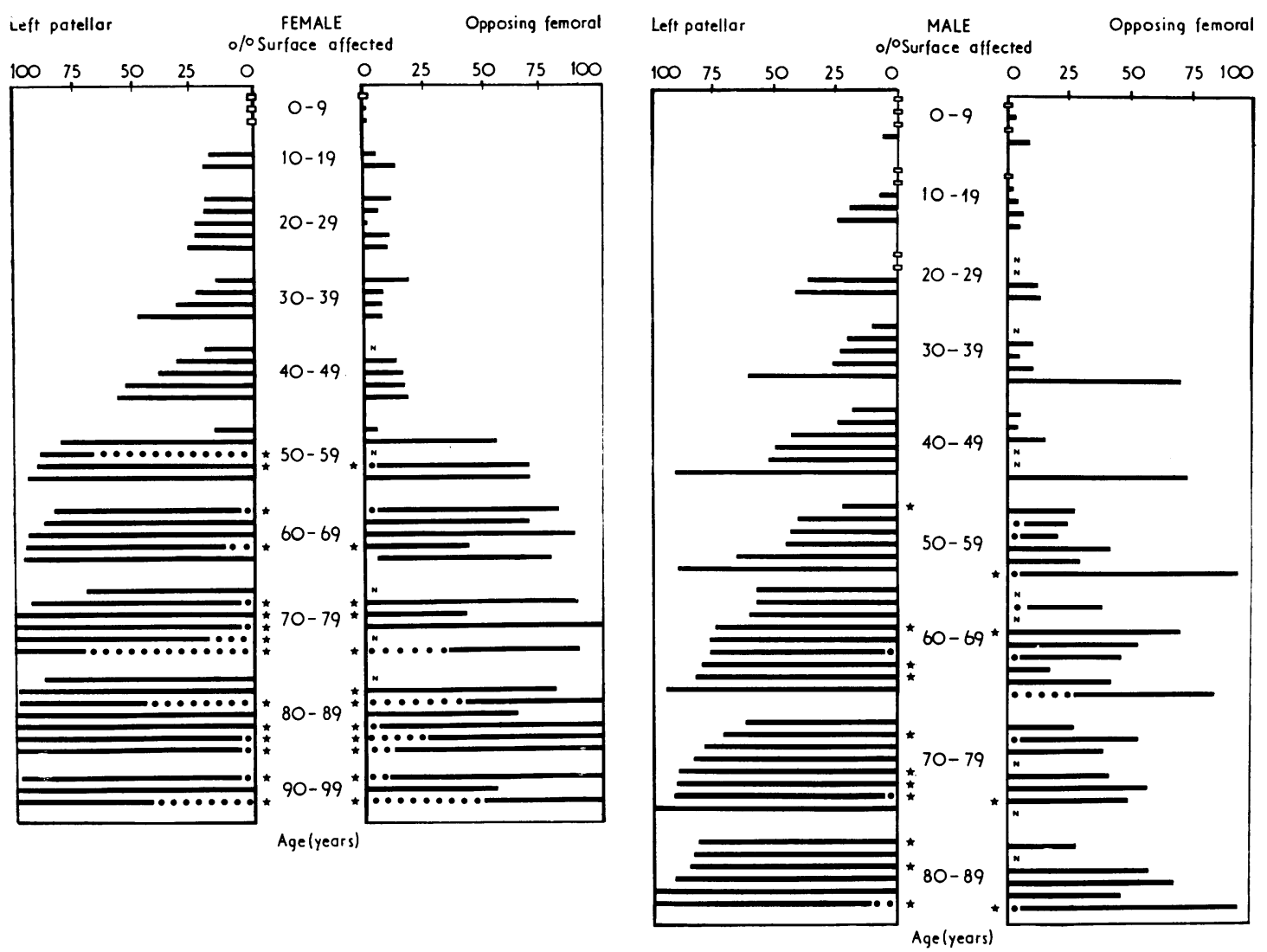

FIGS 5 AND 6 Histograms of individual results for the patello-femoral component of 98 left knee joints, showing the percentage of the articular cartilage surface area affected by overt destructive change; the percentages shown also include sites of peripheral fibrous covering. The results are grouped in age-decades, separately for each sex. Within each age-decade the patellar percentages are arranged in increasing order of magnitude; for each individual the result, where available, for the opposing femoral surface is shown horizontally opposite that for the patella (Key in Fig. 7)

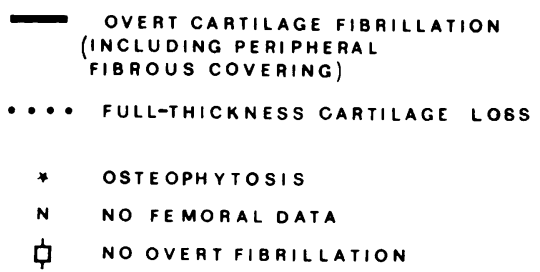

FIG. 7 Key to Figs 5, 6, and 9
Taking the results as a whole, overt changes on the opposing femoral surface tended to develop in parallel with those on the left patellar surface, but, particularly in older men, the percentage area of femoral change tended to be less extensive (Figs 5, 6 , and 8). In both men and women, the total amount of overt surface change was in general similar between the left and right patellae of the same individual (Fig. 9), although it will be noted that a more extensive

Table Total point counts obtained from photographic prints at a magnification of $\times 2$ from a representative selection of patellar articular surfaces from men and women in the age range 20-89 years

\begin{tabular}{|c|c|c|c|c|c|c|}
\hline \multirow[t]{3}{*}{ Sex } & \multicolumn{3}{|l|}{ Left } & \multicolumn{3}{|l|}{ Right } \\
\hline & \multirow{2}{*}{$\begin{array}{l}\text { No. of } \\
\text { specimens }\end{array}$} & \multicolumn{2}{|c|}{ Total points } & \multirow{2}{*}{$\begin{array}{l}\text { No. of } \\
\text { specimens }\end{array}$} & \multicolumn{2}{|c|}{ Total points } \\
\hline & & Mean & Range & & Mean & Range \\
\hline Male & 13 & 172 & $142-212$ & 6 & 176 & 149-224 \\
\hline Female & 20 & 142 & $109-181$ & 7 & 135 & $106-173$ \\
\hline
\end{tabular}

The medial and lateral portions of the surface were photographed separately en face. The counts were made using a grid with the points arrayed at the corners of squares $5 \mathrm{~mm} .^{2}$ in area. 
MALE
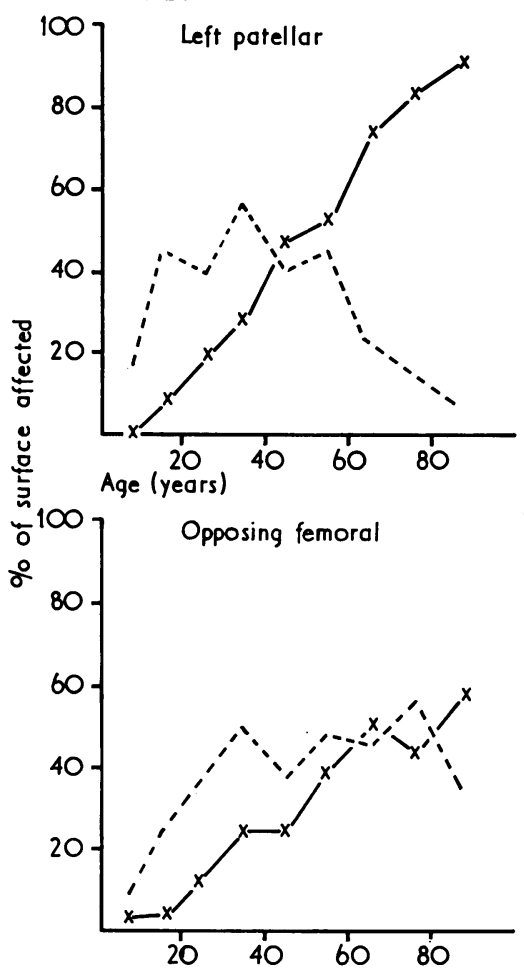

FEMALE

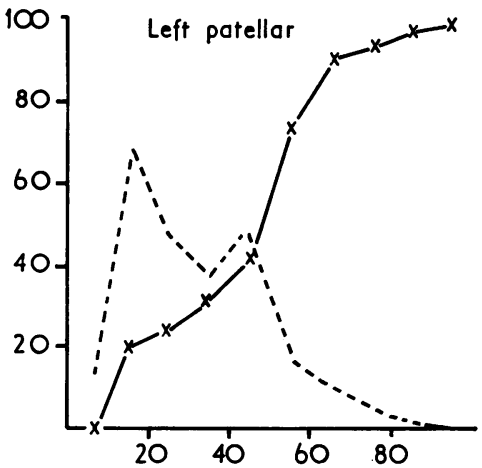

1007 Opposing femoral

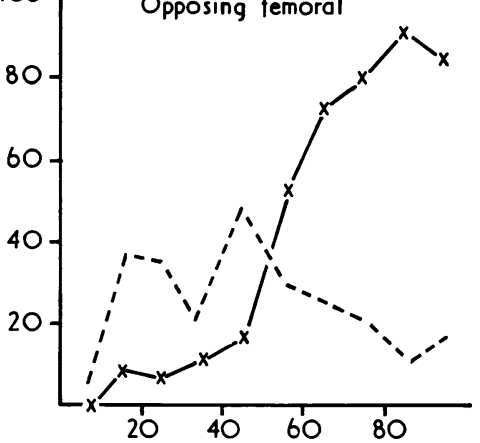

$x-x-x-x$ Overt fibrillation of cartilage \pm full-thickness loss (including peripheral fibrous covering)

. . - Cartilage surface showing dark ink markings against a pale grey background: all patterns

FIG. 8 Graphs of the means for each age-decade of the surface percentages affected by overt destructive cartilage changes and of the surface percentages showing all patterns of dark ink markings against a pale grey background. Sites of peripheral fibrous covering are included with the percentages shown for overt destructive changes

full-thickness cartilage loss was observed on the right side in five of the thirty subjects used for the left-right

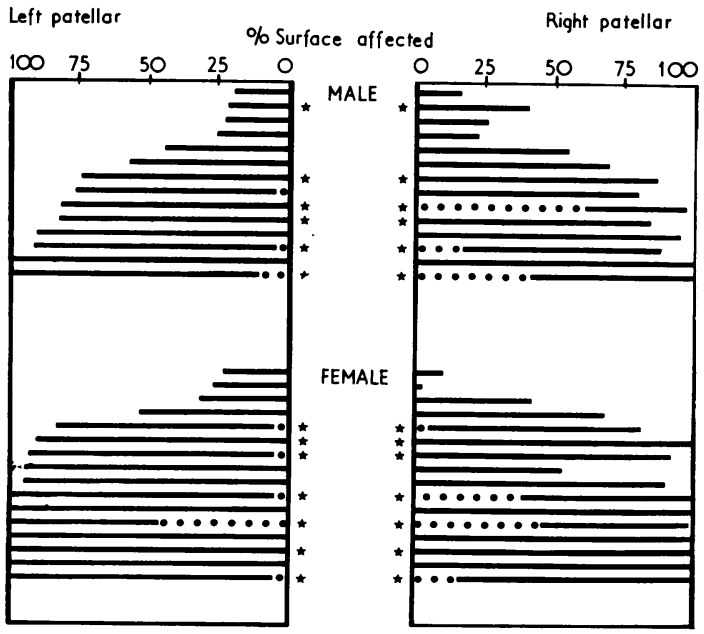
patella comparison.

Full-thickness cartilage loss was not observed in any of the subjects aged 0 to 49 years. In the case of the left patello-femoral articulation in subjects aged 50 or more years, fifteen of 26 women and nine of 29 men showed an area of full-thickness cartilage loss and bone exposure (Figs 3 and 4) on the patellar or opposing femoral or both surfaces (Figs 5 and 6); in eight of the women and in two of the men, the area of full-thickness loss occupied more than 10 per cent. of one or both of the opposing surfaces;

FIG. 9 Histogram of results for the percentage of the patellar articular cartilage surface area affected by overt destructive change compared in the left and right knees of the same individual; the percentages shown also include sites of peripheral fibrous covering. The results are grouped separately for each sex, and arranged in increasing order of magnitude of the left patellar percentages (Key in Fig. 7) 
the figures quoted include those subjects in whom quantitative data was obtained from the patellar surface only. In the case of the right patellar surface in subjects aged 50 or more years, four of twelve women and three of ten men showed an area of fullthickness cartilage loss and bone exposure; in three of the women and in three of the men this area occupied more than 10 per cent. of the right patellar surface.

Marginal osteophytosis (Figs 5 and 6) at the patello-femoral articulation was observed only amongst subjects aged 50 years or more. In the women from this age-range there was mostly a good correlation between the presence or absence of fullthickness cartilage loss on the left patellar or opposing femoral or both surfaces and the presence or absence of marginal osteophytosis on one or both of the opposing faces. In the men, however, the correlation was often poor, this poor correlation being due partly to the presence of minor degrees of osteophytosis on a number of the male patellae from articulations which had no full-thickness cartilage loss on either of the opposing surfaces. For both sexes the presence or absence of marginal osteophytosis on the patella showed a good correlation between the left and right sides (Fig. 9).

In its earlier stages (Fig. 1), overt fibrillation of the patellar cartilage particularly affected the medial part of the medial facet and the periphery of the articular surface, the phenomenon sometimes being accompanied by peripheral fibrous covering. In later stages (Fig. 2), no anatomical site on the patellar surface was immune from damage by overt fibrillation. When there was an area of full-thickness loss of patellar cartilage with bone exposure (Fig. 3), this was usually sited on the lateral and central aspects and did not initially involve the medial part of the medial facet, thus initially sparing one of the main sites first affected by overt fibrillation. Bone exposure on the opposing femoral surface (Fig. 4) was usually sited on the central or on the lateral and central aspects. Further analysis of the topographical distribution of age-related cartilage changes at various stages is the subject of a separate study (Emery and Meachim, unpublished).

\section{PERIPHERAL FIBROUS COVERING}

Vertical sections from a representative series of the adult patellae were examined histologically to investigate the contribution of fibrous covering to the data obtained for confluent or semi-confluent blackening of the surface. A peripheral covering layer of fibrous tissue (Fig. 10) over the cartilage at one, two, or more poles was observed in the majority of the patellae thus examined. Extension of the covering for a distance of more than $1 \mathrm{~mm}$. on to the articular face was infrequent at the proximal, distal and lateral poles; at the medial pole, in contrast,

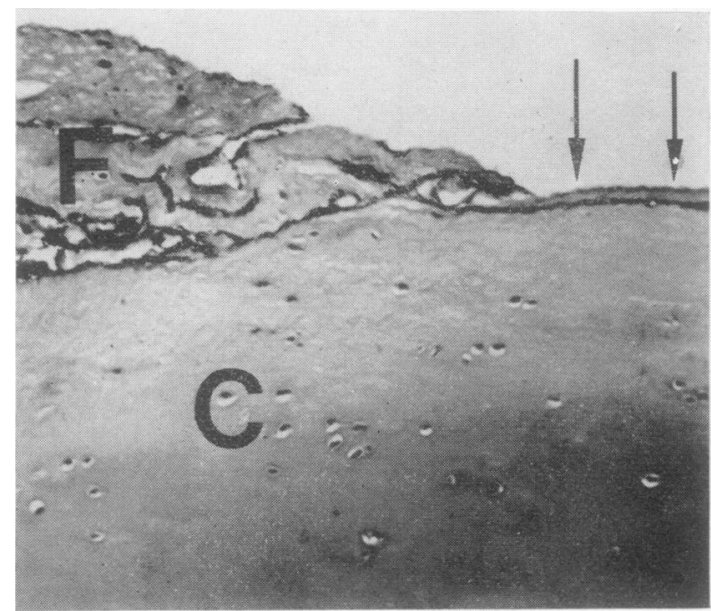

FIG. 10 Photomicrograph of vertically-cut histological section through the periphery of one pole of a patellar articular surface. The cartilage $(C)$ has a covering layer of fibrous tissue $(F)$ with a thin leading edge (arrows). Indian ink preparation. Toluidine blue $\times 150$

extension for distances of from 2 to $6 \mathrm{~mm}$. on to the medial facet was not uncommon, although in other instances no fibrous covering at this pole was apparent. At all poles minor amounts of peripheral covering can occur in the absence of any obvious destructive thinning of the cartilage beneath the fibrous tissue, but at the medial pole a more extensive cover was usually associated with overt destructive changes in the underlying cartilage of the medial facet.

Plaques of fibrous tissue on more central parts of the cartilage surface were sometimes noted at sites where the cartilage was deeply fibrillated or showed full-thickness loss, but otherwise were exceptional.

\section{'Minimal fibrillation'}

The quantitative results will now be presented for surface areas in which the cartilage showed various patterns of dark ink markings against a pale grey background. In adults this appearance can be termed 'minimal fibrillation', since histology of vertical sections shows that in adults it is usually associated with minor degrees of splitting at the cartilage surface (Meachim, 1972a). In children, however, the significance of the appearance is sometimes debatable, since in childhood certain patterns of ink markings can form on surfaces which seem to be essentially intact when subsequently examined histologically in vertical sections at a magnification of $\times 150$ (Meachim and Emery, unpublished). The mean percentage areas showing dark markings of all types, regardless of pattern, are depicted for the left patello-femoral articulation in Fig. 8. It will be seen that the total area showing this 


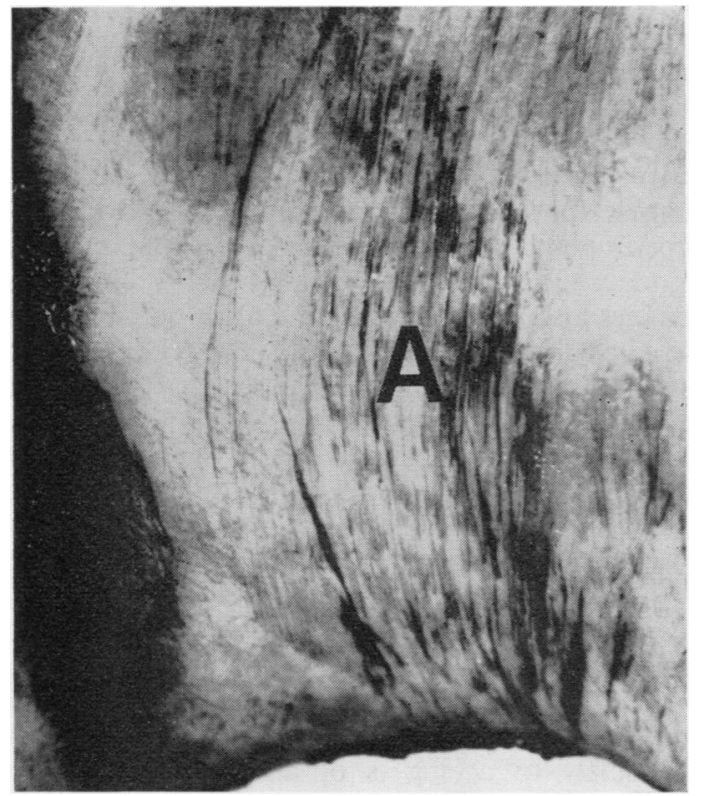

MALE

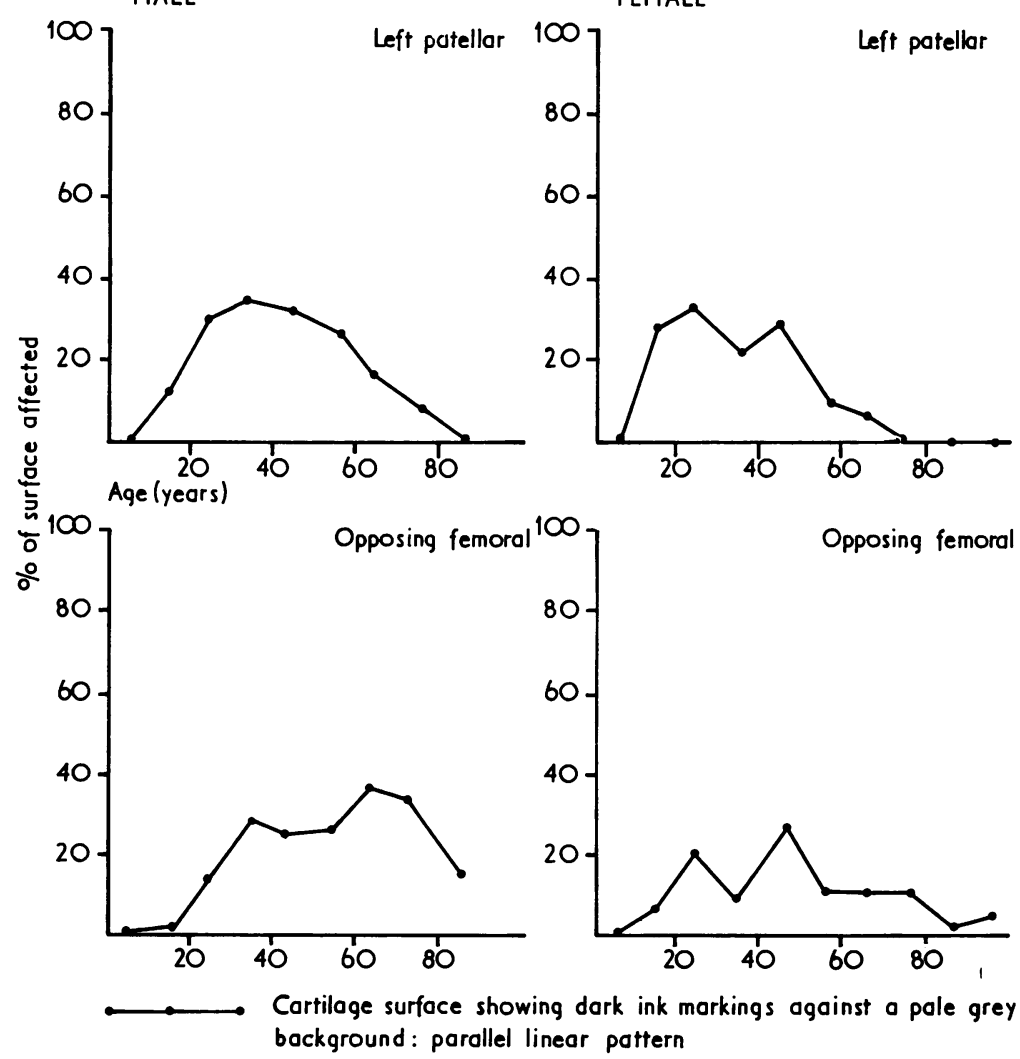

FIG. 11 Part of femoral surface of patello-femoral articulation from a man aged 40 years. Dark markings against a pale grey background $(A)$ are seen running in approximately parallel alignments; an area of confluent blackening is also seen, at the left of the field. Indian ink preparation viewed en face

appearance became extensive during the earlier age-decades; in older subjects, except on the male opposing femoral surface, the area affected gradually decreased, presumably because the sites concerned were replaced or overrun by the extensive development of overt fibrillation.

At the patello-femoral articulation the dark markings showed two main types of patterns:

(1) Sites with macroscopic linear markings in parallel alignments (Fig. 11);

(2) Sites with other, more complex, patterns.

For 86 of the patellar and 83 of the femoral specimens, the quantitative results were further subdivided according to the macroscopic pattern of markings. The mean percentages determined separately for areas showing macroscopic parallel linear markings are depicted for the left patello femoral articulation in Fig. 12. at all ages this pattern of mark? ings is indicative of one type of 'minimal fibrillation'.

FIG. 12 Graphs of the means for each age-decade of the surface percentages showing parallel linear macroscopic patterns of dark ink markings against a pale grey background, an appearance which represents one form of 'minimal fibrillation' of the cartilage surface 


\section{Discussion}

A random series of necropsy subjects may not be representative of comparable age and sex groups amongst the living population. This objection applies particularly to necropsy studies based entirely on patients dying of disease in hospital. In the present study an attempt was made to meet in part this objection by including, as far as practicable, subjects dying from accidents and subjects dying outside hospital suddenly or unexpectedly from natural causes. It may thus be assumed that the present results are reasonably representative of the state of the patello-femoral articulation amongst the population of the Liverpool area. The study was made on white persons only, to permit comparison with Indian data from New Delhi (Roy, Emery, and Meachim, unpublished).

The results indicate that destructive cartilage lesions are extremely common at the patello-femoral articulation amongst Liverpool adults, and that they are often already apparent in teenagers. It is a matter of speculation whether or not children younger than this might develop cartilage surface alterations which later predispose to the onset of actual fibrillation: in this context a distinction has to be made between hypothetical alterations of a potentially harmful nature and alterations attributable simply to cartilage maturation (Meachim, 1969). In adults, the destructive process, subject to variation between individuals in its rate of development, can with ageing progress in the sense of spreading across the articular surface to involve eventually virtually the whole of the patellar and, particularly in women, a considerable proportion of the opposing femoral cartilage; the destructive process can also progress in the sense of vertical spread to give rise to areas of full-thickness cartilage loss with bone exposure amongst some persons aged over 50 years. The average 'rate of progression' in either of these senses is relatively slow in terms of the total life span of an individual.

The present quantitative results from the patellofemoral articulation are in line with the qualitative observations made by Bennett and others (1942) on changes in the knee joint at various ages. Goodfellow and Bullough (1967) have observed similar agechanges in the radio-humeral joint; they noted also that the cartilage of the humero-ulnar joint, in contrast, shows no such pattern of degeneration. Thus the susceptibility of articular cartilage to 'wear' (Radin and Paul, 1972) varies according to site (Meachim, 1969), and would seem to be partly dependent on factors such as the geometrical shape of an articular surface and the type of movement which takes place at the joint concerned (Goodfellow and Bullough, 1967).

The present results are relevant to the question of the pathogenesis of the cartilage lesions in patients presenting clinically with patello-femoral osteoarthrosis. In the case of patients with morphological changes caused by an 'osteoarthritic' factor, such as a mechanical abnormality of the joint, acting independently from the effects of ageing, the results suggest that such lesions are likely often to be superimposed on lesions caused by ageing, and that in many such patients patello-femoral osteoarthritic changes and age changes are unlikely to develop at topographically independent sites. The results, especially those from women, also support the suggestion (Bennett and others, 1942) that in the elderly ageing in itself may alone sometimes cause sufficient patello-femoral damage to constitute osteoarthrosis, since the destructive cartilage lesions observed in the present study would seem, in their advanced form, to be basically similar to those observed in surgically excised femoral heads from patients with clinical evidence of osteoarthrosis of the hip (Meachim, 1972b). This conclusion does not necessarily imply that patello-femoral osteoarthrosis due solely to ageing is of any major numerical importance in terms of its contribution to the total amount of clinically significant patello-femoral osteoarthrosis in the Liverpool population.

The present findings are also relevant to discussions of the pathology of chondromalacia patellae (Øwre, 1936; Wiles, Andrews, and Devas, 1956; Outerbridge, 1961; Bentley, 1970), since they show that the appearance of the patellar articular surface in patients with this clinical condition needs to be assessed in terms of how far it differs quantitatively or qualitatively from that in specimens matched by age and sex from amongst the general population.

\section{Summary and conclusions}

A quantitative study of destructive cartilage changes in the patello-femoral component of the knee joint has been made in a random series of necropsies in Liverpool on 98 white subjects (53 male; 45 female) whose ages ranged from 4 weeks to 94 years. Data obtained by point-counting of indian ink preparations of the patellar and of the opposing femoral articular surfaces was analysed separately for each age-decade and for each sex.

The results indicate that overt destructive cartilage changes are extremely common at the patellofemoral articulation amongst Liverpool adults, and that such changes are often apparent in teenagers. Within each age-decade there is variation between individuals in the percentage area of overt fibrillation, and, for older subjects, in the percentage area of full-thickness cartilage loss and in the presence or absence of osteophytosis at the articular margin. With increasing age, overt destructive change, subject to the variation from individual to individual 
in its rate of development, sooner or later involves virtually the whole of the patellar and, particularly in women, a considerable proportion of the opposing femoral surface. Although its average rate of development is relatively slow in terms of the expected total lifespan of an individual, the destructive process can give rise to areas of full-thickness cartilage loss with bone exposure amongst some persons aged over 50 years. These observations on necropsy material are discussed in relation to patients presenting clinically with patello-femoral osteoarthrosis.

We are grateful to Dr. M. J. Bouton, Dr. J. Burns, Dr. P. H. Buxton, Dr. E. Glazowski, Dr. M. J. J. Wodzinski, and Dr. A. S. Woodcock for their co-operation during the collection of specimens.

\section{References}

Bennett, G. A., Waine, H., ANd Bauer, W. (1942) 'Changes in the Knee Joint at Various Ages with Particular Reference to the Nature and Development of Degenerative Joint Disease'. Commonwealth Fund, New York

Bentley, G. (1970) J. Bone Jt Surg., 52A, 221 (Chondromalacia patellae)

Bullough, P. G., AND Goodfellow, J. W. (1968) Ibid., 50B, 852 (The significance of the fine structure of articular cartilage)

Byers, P. D., Contepomi, C. A., AND Farkas, T. A. (1970) Ann. rheum. Dis., 29, 15 (A post-mortem study of the hip joint)

Collins, D. H. (1949) 'The Pathology of Articular and Spinal Diseases', pp. 74-115. Arnold, London

Goodfellow, J. W., AND Bullough, P. G. (1967) J. Bone Jt Surg., 49B, 175 (The pattern of ageing of the articular cartilage of the elbow joint)

HeINE, J. (1926) Virchows Arch. path. Anat., 260, 521 (Über die Arthritis deformans)

Kellgren, J. H. (1961) Brit. med.J., 2, 1 (Osteoarthrosis in patients and populations)

LAST, R. J. (1972) 'Anatomy, Regional and Applied', 5th ed., p. 245. Churchill Livingstone, Edinburgh and London

MeACHIM, G. (1969) Clin. Orthop., 64, 33 (Age changes in articular cartilage)

- (1972a) Ann. rheum. Dis., 31, 457 (Light microscopy of indian ink preparations of fibrillated cartilage)

- (1972b) J. Path., 107, 199 (Articular cartilage lesions in osteoarthritis of the femoral head)

OUTERBRIDGE, R. E. (1961) J. Bone Jt Surg., 43B, 752 (The aetiology of chondromalacia patellae)

ØWRE, A. (1936) Acta chir. scand., 77, Suppl. 41 (Chondromalacia patellae)

Radin, E. L., AND PAUL, I. L. (1972) J. Bone Jt Surg., 54A, 607 (A consolidated concept of joint lubrication)

WALMSLEY, R. (1972) In 'Cunningham's Textbook of Anatomy', 11 th ed., ed. G. J. Romanes, p. 241. Oxford University Press, London

Wiles, P., ANDrews, P. S., ANd Devas, M. B. (1956) J. Bone Jt Surg., 38B, 95 (Chondromalacia of the patella) 